\title{
ESTUDIANTES MIGRANTES EN LA ADAPTACIÓN DE LA EDUCACIÓN COLOMBIANA
}

\section{MIGRANT STUDENTS IN THE ADAPTATION OF COLOMBIAN EDUCATION}

\section{Contreras Manrique Rocío de Belén ${ }^{1}$}

Olga Lucy Rincón Leal ${ }^{2}$

\section{Contreras Manrique Liliana ${ }^{3}$}

Universidad Pamplona

\section{RESUMEN}

Es relevante en el proceso de adaptación escolar colombiano con los estudiantes migrantes de la zona de frontera del corregimiento de la parada, identificar los factores que influyen en su inteligencia emocional. La investigación considero resaltar los factores que influyen en la inteligencia emocional como atención o percepción, comprensión y regulación del ser humano. Por consiguiente, se aplicó el cuestionario como herramienta de estudio la escala TMMS-24, de Salovey y Mayer $^{1}$, con un diseño descriptivo cuantitativo, muestra

\footnotetext{
$1 \quad$ Maestría en prácticas pedagógicas, Universidad de Pamplona: rocio_de_belen@yahoo.com.mx; ORCID: https://orcid.org/0000-0002-4434-0408. Grupo C/COM

2 Maestría en Educación Matemática. Universidad Francisco de Paula. (Colombia). ORCID: orcid.org/00000002-8080-496X. olgarincon@ufps.edu.co Grupo EULER 3 Psicóloga y Mg en Orientación, Universidad Pamplona: lilianacontrerasmanrique@yahoo.com.mx; ORCID: https://orcid.org/0000-0002-8586-2093
}

100 migrantes. Por lo tanto, la educación colombiana tiene el compromiso de orientar a los educandos. Resultados: un $60 \%$ de los estudiantes migrantes tienen la percepción o atención y un $40 \%$ siente ansiedad e inseguridad al vivir en otro país. Se evidenció que en el factor de claridad emocional un $80 \%$ de los estudiantes comprende sus sentimientos y razona emocionalmente. Los resultados demuestran que un $50 \%$ tiene dificultades en regular o reparar los estados emocionales correctamente En conclusión, los factores que presentan dificultad son los de atención o percepción, así como el factor reparación o regulación de las emociones, dado que representan otra cultura y normatividad en ámbito social - educativo Colombiano. 


\section{PALABRAS CLAVE}

Inteligencia emocional, niveles de percepción, comprensión, regulación de las emociones, adaptación

\section{ABSTRACT}

It is relevant in the Colombian school adaptation process with migrant students from the border area of the town of La Parada, to identify the factors that influence emotional intelligence in migrant students. The research, I consider, highlight the factors that influence emotional intelligence such as attention or perception, understanding and regulation of the human being. Therefore, the questionnaire was applied as a study tool, the TMMS-24 scale, by Salovey and Mayer. , with a descriptive quantitative design, it shows 100 migrants. Therefore, Colombian education is committed to guiding students. Results; $60 \%$ of migrant students have the perception or attention and $40 \%$ of migrant students feel anxiety and insecurity living in another country. It was evidenced, $80 \%$ of migrant students, in the factor of emotional clarity, in which they understand their feelings and reason emotionally; finally, the repair factor, the ability to regulate one's own and other people's emotions with the level of regulation, the results show, $50 \%$ of migrant students have difficulties in regulating or repairing emotional states correctly I conclude: the factors that present difficulty Migrant students are the factors of attention or perception and the factor of repair or regulation of emotions, since it represents another culture and regulations in the Colombian social-educational sphere.

\section{KEY WORDS}

Emotional intelligence, levels of perception, understanding, regulation of emotions, adaptation

\section{RESUMO}

É relevante no processo de adaptação escolar colombiana com estudantes migrantes da zona fronteiriça da cidade de La Parada, identificar os fatores que influenciam a inteligência emocional em estudantes migrantes. As pesquisas, considero, destacam os fatores que influenciam a inteligência emocional, como atenção ou percepção, compreensão e regulação do ser humano. Portanto, o questionário foi aplicado como ferramenta de estudo, a escala TMMS-24, de Salovey e Mayer. , com desenho quantitativo descritivo, mostra 100 migrantes. Portanto, a educação colombiana tem o compromisso de orientar os alunos. Resultados; $60 \%$ dos estudantes migrantes têm a percepção ou atenção e $40 \%$ dos estudantes migrantes sentem ansiedade e insegurança por viver em outro país. Evidenciou-se, $80 \%$ dos alunos migrantes, no fator clareza emocional, em que compreendem seus sentimentos e raciocinam emocionalmente; Por fim, quanto ao fator de reparação, a capacidade de regular as emoções próprias e alheias com o nível de regulação, os resultados mostram que $50 \%$ dos estudantes migrantes têm dificuldade em regular ou reparar os estados emocionais corretamente. Concluo: os fatores que apresentam dificuldade Os estudantes migrantes são os fatores de atenção ou percepção e o fator de reparação ou regulação das emoções, já que representam outra cultura e normas no âmbito socioeducativo colombiano.

\section{PALAVRAS-CHAVE}

Inteligência emocional, níveis de percepção, compreensão, regulação das emoções, adaptação 


\section{RÉSUMÉ}

II est pertinent dans le processus d'adaptation scolaire colombien avec les étudiants migrants de la zone frontalière de la ville de La Parada, d'identifier les facteurs qui influencent l'intelligence émotionnelle des étudiants migrants. La recherche, je considère, met en évidence les facteurs qui influencent l'intelligence émotionnelle tels que l'attention ou la perception, la compréhension et la régulation de l'être humain. Par conséquent, le questionnaire a été appliqué comme un outil d'étude, l'échelle TMMS-24, par Salovey et Mayer., avec une conception quantitative descriptive, il montre 100 migrants. Par conséquent, l'éducation colombienne s'est engagée à guider les étudiants. Résultats; $60 \%$ des étudiants migrants ont la perception ou l'attention et $40 \%$ des étudiants migrants ressentent de l'anxiété et de l'insécurité en vivant dans un autre pays. II a été mis en évidence, $80 \%$ des étudiants migrants, dans le facteur de clarté émotionnelle, dans laquelle ils comprennent leurs sentiments et raisonnent émotionnellement; Enfin, le facteur de réparation, la capacité à réguler ses émotions et celles des autres avec le niveau de régulation, les résultats montrent que $50 \%$ des étudiants migrants ont des difficultés à réguler ou à réparer correctement les états émotionnels. Je conclus: les facteurs qui présentent des difficultés Les étudiants migrants sont les facteurs d'attention ou de perception et le facteur de réparation ou de régulation des émotions, car ils représentent une autre culture et réglementation dans la sphère socio-éducative colombienne.

\section{MOTS CLÉS}

intelligence émotionnelle, niveaux de perception, compréhension, régulation des émotions, adaptation

\section{INTRODUCCIÓN}

Actualmente en la zona de frontera entre Colombia y Venezuela en el corregimiento de la Parada del municipio de Villa Rosario, transitan 2.500 a casi 38.000 individuos de las diferentes etapas del ciclo vital ; ya que se presentan dificultades socio-económicas-educativas con índices de desempleo siendo así afectada la economía de la familia; por lo tanto, unas de las causas del gran impacto en los migrantes venezolanos, es el entorno social, en la cual se han generado cambios socio-familiar-educativos en la de frontera.

Diario La Opinión. (2019). Las estadísticas que maneja Migración Colombia, según el registro administrativo de migrantes venezolanos en Colombia, es que en Villa del Rosario el $23 \%$ de su población son migrantes irregulares. Dobló su población en apenas cuatro años, tras el cierre de frontera. De los 38.000 migrantes que entran a Colombia por el corregimiento de la Parada, unos 8.000 se quedaron a vivir en este barrio, el resto va y viene por sus calles en una romería sin fin que también demanda servicios de transporte y agua, esto último el eterno problema en Villa del Rosario. Por sus calles es rutina ver hombres y ahora también mujeres, de día y noche, cargando pesados bultos con contrabando: carne, comida, gaseosas.

El proceso de adaptación en los educandos migrantes es importante en el contexto escolar colombiano, siendo eje central en los estudiantes el comportamiento emocional y apreciándose en el comportamiento los factores de percepción, comprensión y regulación de las emociones. Por otra parte, es conveniente que en los colegios realicen estrategias para rechazar antivalores como el acoso escolar y la xenofobia para la transformación personal, en la cual ayude a la adaptación de los estudiantes migrantes en su nuevo contexto social. 
En el año 2020; todos los días 2.535 niños cruzaban la frontera de Venezuela a Colombia para asistir a clases. Unos cruzan Puerto Santander- y otros el Puente Internacional Simón Bolívar. Estos niños tenían apoyo de un corredor humanitario que les permitía un trato especial. A consecuencia de la pandemia o el coronavirus se modifico todo, siendo crítica la situación para los estudiantes, maestros y padres de familia.

En noticia Caracol- radio. (2020). En la zona de frontera se pondrá en marcha un plan piloto para estudiantes venezolanos que buscará ser replicado por las demás instituciones educativas de la ciudad. Silva Pablo (2020); rector del colegio Misael Pastrana, dijo a Caracol Radio que "se hizo la entrega de las primeras guías de estudio; hay guías para dos semanas, contienen la evaluación de los procesos, los padres de familia tienen una red de distribución y de captación que llegarán a cada uno de los estudiantes que viven en Venezuela". La idea del corredor humanitario, según explicó la secretaria de Educación de Norte de Santander, Laura Cáceres Niño, es lograr llevar a los niños el material pedagógico

El Consejo Noruego para Refugiados ha estado liderando la iniciativa de reactivar el corredor humanitario junto a las autoridades educativas. Nunca en el mundo tantos niños y niñas habían estado incomunicados con sus escuelas o habían interrumpido por completo su aprendizaje. Por eso necesitamos encontrar opciones solidarias para que niños y niñas, que viven en Venezuela y que estaban estudiando en Colombia, accedan a materiales educativos y puedan retomar su educación. Estos materiales les ayudarán a los menores de edad a mejorar sus vidas, brindarán esperanza y los mantendrán a salvo en sus hogares", dijo a Semana Yadira Galeano, gerente de área del consejo noruego para refugiados.
Una gran responsabilidad está en las manos de los educadores, quienes tienen la oportunidad de compartiry realizar la integración en la comunidad educativa (profesores, estudiantes, padres de familia y psicorientador), por lo que habrá un efecto positivo multiplicador en la sociedad. Es importante el estudio en los estudiantes inmigrantes en el proceso de la adaptación escolar colombiana. Para caracterizar los niveles de la inteligencia emocional a partir de la aplicación del cuestionario TMMS 24 en los estudiantes inmigrantes en el proceso de adaptación escolar colombiana. Se hace necesario que la educación colombiana analice y oriente a los educandos para que la educación sea proceso educativo, continuo y permanente, en la cual logre el desarrollo integral del educando.

El teórico Goleman (2000) o Bar-On (2010). Comparo entre inteligencia y competencia emocional resaltando que la inteligencia emocional es el centro de las habilidades para razonar con las emociones mientras la competencia emocional es cuando el individuo ha alcanzado un nivel determinado de logro emocional. ${ }^{4}$ Se implementó los tres factores de la Inteligencia emocional que mide TMMS-24, Ramos, Enríquez, y Recondo. (2012), se utilizó en el presente estudio como la "Percepción emocional: La persona es capaz de sentir y expresar los sentimientos. comprensión de sentimientos: comprende las emociones. Regulación emocional: regula las emociones". El objetivo general. Se identifico los factores que influyen en la inteligencia emocional en los estudiantes migrantes a través de la escala TMMS-24 en el proceso de adaptación escolar colombiana y los objetivos especifico; seguidamente, se establecio los tres factores de la inteligencia emocional en los estudiantes migrantes en el proceso de adaptación escolar colombiana y se caracterizo el comportamiento emocional en los tres niveles de la inteligencia 
emocional a través del test TMMS-24 en los estudiantes migrantes en el proceso de la adaptación escolar colombiana y por último se caracterizo los tre factores incluyente en la inteligencia emocional en los estudiantes migrantes. Por consiguiente; "Durante el primer semestre del 2020 han llegado a nuestro país un poco más de 234 mil ciudadanos venezolanos. Si miramos el comportamiento de los últimos tres meses, podríamos decir que, diariamente, esa cifra es cercana a los mil seiscientos registros y a medida que se agudice la situación en Venezuela, el flujo continuará en aumento", afirmó el director de Migración Colombia, Christian Krüger

La Secretaría de Educación Municipal de San Josè de Cùcuta solicitó incluso al Ministerio de Educación un trato distinto en los parámetros de valoración, debido a que es una de las ciudades que ha recibido a más alumnos venezolanos. EI año pasado la cifra se ubicó en aproximadamente 4.000 , y este año ya está en 9.700 alumnos.

Es relevante la Implementación de encuentros formativos para hacer una transformación personal y rechazar el bullying y la xenofobia. Por lo tanto, la carencia de amor, la amistad y el apoyo por parte de los estudiantes colombianos afectan el proceso de aprendizaje y la adaptación en el estudiante inmigrante; siendo el factor problema el cambio de cultura, las tradiciones, el lenguaje, siendo el eje central del problema la interculturalidad y los estilos de aprendizaje. Transformando la problemática que viven los estudiantes inmigrantes es necesario educar en valores sobre la inmigración para desarrollar el respeto y la valoración hacia el otro; por lo tanto, acabar con la estigmatización o desvalorización en los inmigrantes venezolanos ya sea a nivel educativo y social.

Por esta razón, se hace referencia la educación de calidad sin barreras. Los países deben garantizar la educación con equidad e inclusivo, para ser una plena realización humana con la adaptabilidad y estabilidad socioeducativo generando así la paz con los lazos de hermanda para la población migrante y que no sea afectada al ingresar en la educación pùblica. (UNESCO, 2017, P7).

Es notable que los niños $y$ adolescentes migrante el derecho a la educación, corresponde señalar que el estado colombiano debe apoyar - garantizar e implementar las políticas para permitir el acceso a la educación, salud y alimentación, según lo expresado por la UNESCO 2019.

En la Corte Constitucional en la sentencia SU677 de 2017 en los derechos de los niños, niñas y adolescentes migrantes advierte:

\section{Losprincipioscrelacionadosconelreconocimiento} de los niños, niñas y adolescentes como sujetos, se encuentra en la Convenciòn Internacional sobre los derechos del niño y son (i)la igualdad y no discriminación; (ii) el interés superior de las y los niños; (iii) la efectividad y prioridad absoluta; y (iv) la participación solidaria. Por tal motivo la convención internacional sobre derechos del niño y la Constitución Política de Colombia, las y los niños no sólo son sujetos de derechos, sino que sus derecho e intereses prevalecen en nuestro ordenamiento Jurídico.

El plan nacional de desarrollo "Pacto por Colombia, pacto por la equdad (2018 -2022); se creo la estrategia nacional de acceso, bienestar y permanencia, desde la gestión de la matrícula y la oportunidad de acompañamiento a la pertenencia e integra alternativa para la mejora de la convivencia escolar y efectiva inclusión educativa a los niños y adolescentes migrantes provenientes de Venezuela.

\section{PROBLEMA DE INVESTIGACIÓN}

La pregunta de la investigación. ¿Cuáles son los factores que influyen en la inteligencia emocional en los estudiantes migrantes para el proceso de la adaptación escolar colombiana?. 


\section{MARCO TEÓRICO}

El estudio se apoya en el teórico Goleman (2000) o Bar-On (2010), el teórico, menciono, la diferencia entre inteligencia y competencia emocional afirmando que la inteligencia emocional representa el eje central de las habilidades para razonar con las emociones, por lo tanto, la competencia emocional la persona alcanza el nivel del logro emocional.

De acuerdo; con Ramos, Enríquez, y Recondo, (2012), las tres dimensiones de la Inteligencia emocional que mide TMMS-24, que se utilizará en el presente estudio son: "Percepción emocional: Cuando la persona es capaz de sentir y expresar los sentimientos con autocontrol. Comprensión de sentimientos: es comprender y analizar las emociones. Regulación emocional: Cuando una persona es capaz de regular las emociones". ( $p$. 45).

Ruiz Monterroso, Ileana Ninette (2014). Los niveles de las dimensiones de inteligencia emocional en los estudiantes de primeros semestres del programa de psicología clínica e industrial de la jornada vespertina de la Universidad Rafael Landívar. Con una muestra de 152 , se pudo concluir que no alcanzaron significancia, la dimensión que heterogénea fue la regulación emocional.

Bustos, Raùl y Gairín Joaquín (2017). Adaptación académica de estudiantes migrantes en contexto de frontera. En Chile. Los autores analizarón las características asumidas por el proceso de adaptación académica de los estudiantes migrantes incorporados a la educación básica y media de la ciudad de Arica, Chile, contexto de investigación caracterizado por su situación bifronteriza. Las entrevistas se aplicaron a jefes de unidades técnico-pedagógicas, y a estudiantes migrantes. En este estudio se visualiza que estudiante se adapta a la cultura del colegio, desarrollando procedimientos normalizadores. Dentro de la norma se han adaptado los estudiantes como producto a este mecanismo se obtiene buenos rendimiento escolar.

Salas, N., Castillo, D., San Martín, C., Kong, F., Thayer, E., \& Huepe, D. (2017). migración en la escuela: caracterización del prejuicio hacia escolares migrantes en Chile, con los aspectos asociados al prejuicio existente hacia los migrantes en el sistema escolar y ejecutando propuesta por la psicología social, y brindando orientaciones para potenciar la inclusión de la diversidad cultural en el sistema educacional, tanto a nivel de contexto, de políticas como de prácticas pedagógicas.

Sosa Fernanda Mariel y Zubieta Elena. (2015). La experiencia de migración y adaptación sociocultural: identidad, contacto y apoyo social en estudiantes universitarios migrantes. Contribuyeròn a una mejor adaptación sociocultural y psicológica, en este artículo de investigación, exploraron los niveles que los estudiantes exhiben en aspectos relacionados con la experiencia de migración: identificación con el endogrupo y exogrupo, nivel de contacto y apoyo social percibido, y grado de adaptación sociocultural. Con una muestra de 214 estudiantes migrantes de ambos sexos de universidades públicas y privadas de Buenos Aires. Los estduante han sido recptores con mayor contacto y percepción de apoyo social considerados de adaptación sociocultural. Se registran diferencias en función del origen interno o externo del migrante.

Ferrer, Rauqel y otros. (2013). Proceso de aculturación y adaptación del migrante. La migración implica una serie de procesos cognitivos, motivacionales $y$ afectivos que afectan su adaptación. Sin embargo es importante las estrategias de afrontamiento e intervención dirigidas a estos colectivos que permitieron analizar los factores individuales como socioculturales, asociados a los procesos de adaptación y aculturación, que describen los 
procesos de aculturación: los que se centran en el contenido (sentimientos, actitudes y conductas), los que se centran en el proceso (establecimiento de relaciones entre inmigrantes y autóctonos) y los interactivos o ampliados, que combinan los dos anteriores.

Castillo, Dante, Santa-Cruz, Eduardo, \& Vega, Alejandro. (2018). Estudiantes migrantes en escuelas públicas chilenas. Analizaron el proceso de escolarización de los niños migrantes, sobre la base de las interrelaciones observadas entre los estudiantes, con el objeto de proporcionar insumos que fortalezcan la dinámica inclusiva del sistema escolar chileno, los resultados muestran la compleja red de representaciones sociales y escolares asociadas con la migración y la permanencia en el sistema escolar formal, así como las dificultades de inclusión en las dinámicas cotidianas de juego y trabajo en espacios escolares. Por tal motivo, es importante que las instituciones escolares tengan políticas específicas que organicen y estructuren la inclusón en la educación.

Bastian Carter Thuillier, Víctor Manuel López Pastor, Francisco Javier Gallardo Fuentes. (2017). Los autores, analizaron la integración del alumnado migrante que participa del Programa Integral de Deporte Escolar del Municipio de Segovia, España (PIDEMSG). El análisis de contenido se ha desarrollado utilizando un sistema de categorías analíticas. Los resultados muestran que el PIDEMSG favorece la integración e inclusión social del alumnado inmigrante, así como la comunicación intercultural y el desarrollo de valores educativos, por su enfoque pedagógico.

Franco García, Martha Josefina. (2014). Los estudiantes migrantes: sujetos emergentes del derecho a la educación. analizo la presencia de estudiantes inmigrantes que llegan a las escuelas de educación básica en México. Estos niños migrantes mexicanos. El trabajo debatio el derecho a la educación.
Bustos-González, R. (2016). estrategias de adaptación académica en estudiantes migrantes de establecimientos de enseñanza ciudad de arica. Analizo las estrategias utilizadas para promover la adaptación académica de los estudiantes inmigrantes incorporados en la educación básica y media de la ciudad de Arica.

Este tipo de investigación busca "universales concretos" alcanzados a través del análisis profundo de casos particulares y de la comparación de ese caso con otros. Por esta razón se opta por un enfoque etnográfico, en virtud de apreciar la realidad como una construcción que solo es posible de conocer, a partir de los significados entregados por los sujetos que la construyen. Los resultados de la investigación señalan que la tradición cultural chilena, muestra que su predisposición a la interculturalidad es limitada. Las escuelas parten de la premisa que es el estudiante quien debe adaptarse al sistema escolar. Así, los mecanismos diseñados para apoyar al estudiante migrante se refieren principalmente al apoyo administrativo. Además, se constata la creencia arraigada en que la situación se "normalizará con el tiempo", lo que en la práctica se traduce en inmovilidad.

Mondaca, Carlos, Muñoz, Wilson, Gajardo, Yeliza, \& Gairín, Joaquín. (2018). Estrategias y prácticas de inclusión de estudiantes migrantes en las escuelas de Arica y Parinacota, frontera norte de Chile. analizarón cómo el sistema educacional formal no ha desarrollado mecanismos institucionales de integración sociocultural que permitan una adecuada inserción de los estudiantes de origen extranjero en las escuelas chilenas; por lo cual, diversos agentes sociales (estudiantes, padres) han debido desplegar estrategias y prácticas de inserción adaptadas al contexto local, generando mecanismos emergentes de integración sociocultural. 
Miranda Asencio Camilo Sebastián. (2019). Percepción de inclusión escolar que tienen los estudiantes migrantes de educación secundaria de establecimientos educacionales municipales en la ciudad de Concepción. universidad del desarrollo. facultad de gobierno magíster en Políticas Educativas. Concepción. La presente investigación busco poner en conocimiento la percepción de inclusión escolar que tienen los estudiantes en calidad de migrantes que ingresan al sistema educacional chileno, poniendo énfasis en las prácticas inclusivas que tiene cada establecimiento educacional para recibir a los alumnos de diferentes nacionalidades.

Mondaca Rojas, Carlos Enrique. (2018). Educación y migración transfronteriza en el norte de chile: procesos de inclusión y exclusión de estudiantes migrantes peruanos y bolivianos en las escuelas de la región de arica y parinacota. Presentando poca atención a las necesidades de aprendizajes de los educandos desde la política pública, excluyendo a los estudiantes culturalmente distintos, al asimilar a todas y todos en aulas saturadas de símbolos y contenidos nacionalistas. existiendo fuerte resistencia en el ámbito político y educacional con la interculturalidad en el la educaciòn urbana, siendo discursos o representaciones esencialistas de la inclusión, pero se presenta maltrato por raza.

Cazzetta, Hannah María. Desafíos para los estudiantes venezolanos en Colombia. (2019). Los conflictos económicos, políticos y sociales que ocurren en Venezuela han provocado que más de millones de venezolanos escapen a países de América Latina. De estos más de dos millones de personas muchos son estudiantes y profesionales recién egresados, incluidos académicos que ya no pueden costear sus estudios o enseñar.

Manzo Álvarez, Claudia Libertad. Perspectivas y prácticas vinculadas a inclusión presentes en la evaluación de desempeño de establecimientos y sus sostenedores: Análisis cualitativo de informes de visitas evaluativas. (2018). La Ley de Inclusión, inscrita en una narrativa de desegregación tendiente a promover mixtura social en las escuelas, convive con otras regulaciones que se encuentran en tensión con estos objetivos, como la Ley 20.529, que dispone la categorización de escuelas y la evaluación de desempeño escolar. El análisis permitio evidenciar la carencia de un entramado discursivo en sustentar, reconocer y evaluar prácticas orientadas a inclusión social, dado el predominio del enfoque de rendición de cuentas y las perspectivas centradas en el déficit.

Castillo Dante y otros. (2016). Programa Interdisciplinario de Investigaciones en Educación -PIIE. María Luisa Santander, Providencia -Santiago. analizaron el proceso de escolarización en educación básica de niños inmigrantes, con el objeto de proveer insumos para fortalecer las dinámicas de inclusión en el sistema escolar chileno. Demostrando el complejo entramado de representaciones sociales y escolares asociadas a la migración en la permanencia en el sistema escolar formal, se concluyo la inexistencia de una política nacional que oriente la práctica cotidiana de las escuelas. Las recomendaciones en orden a establecer una política educativa de carácter nacional que aborde la complejidad del fenómeno, tanto a nivel del sistema educativo, de los sostenedores $y$ de las propias escuelas.

Beniscelli, Leonora, Riedemann, Andrea, \& Stang, Fernanda. (2019). Multicultural, sin embargo, asimilacionista. La sostenida inmigración latinoamericana y caribeña que ha venido experimentando Chile, este artículo presento un estudio de caso de un establecimiento que, desde inicios del mencionado fenómeno migratorio, ha recibido alumnos migrantes, siendo hoy una de las escuelas públicas con las más altas tasas de matrícula extranjera a nivel 
nacional. Esta paradoja (heterogeneidad del alumnado junto a prácticas homogeneizantes) representa un obstáculo en el avance hacia una educación pública que aborde la diversidad cultural presente en las aulas.

Contreras-Manrique, R. de B., ContrerasManrique, L.., Ovalle-Lizcano, T. V.., EspinosaBohórquez, L. M., Valero-Bencardino, J., Coronel-Peñuela, D. L., \& Cabrera-Reyes, A. (2020). Descripción de los problemas sociales influyentes en la transición moral en una sociedad cambiante a través del enfoque cuantitativo. Analizaron las diversas crisis que ha tratado de sobrevivir como la sobrepoblación, las pandemias que reducen la durabilidad, la migracióm, que inducirán al derecho a dar una vuelta y acoplarse a la civilización, llegará el tiempo, que el deber del estado ya no será asegurar la vida sino la supervivencia, en consecuencia, a un control de integrantes en la familia; siendo la moral y el derecho piezas moldeables a las necesidades de la sociedad.

Suárez, A; Espinel, M; Contreras Manrique, L; Contreras Manrique, R. (2015). Herramientas de apoyo para el aprendizaje de los estudiantes que presentan limitaciones auditivas. Identifico las herramientas que brindan un mejor acceso al mundo del aprendizaje y en la inclusión escolar para mejorar la satisfacción personal, comunicación, interacción y los resultados académicos; logrando con esta dinámica bajar los niveles de ansiedad, sentimientos de tristeza e introversión en los pares que presentan discapacidad auditiva, visual, motora en los niños y jóvenes migrantes.

Aravena Kenigs, Omar, Riquelme Bravo, Paula, Mellado Hernández, Ma Elena, \& Villagra Bravo, Carolina. (2019). Inclusión de Estudiantes Migrantes en la Región de La Araucanía, Chile: Representaciones desde los Directivos Escolares. Describio las representaciones de docentes directivos acerca de la inclusión de estudiantes migrantes en centros educativos. por tal motivo, Se destacan la necesidad de transitar hacia liderazgos más inclusivos y críticos, capaces influenciar en favorecer el proceso de enseñanza-aprendizajes desde un enfoque inclusivo e intercultural.

Ovalle Lizcano, T. V, Coronel Peñuela, D. L., Contreras Manrique, R. de B, \& Cabrera Reyes, A. (2019).

Impacto sobre la seguridad personal frente. A la regulación de la ciberdelincuencia en la Universidad

de Pamplona, sede de Villa del Rosario. Es relevante que brinden información y orientación a los

educandos migrantes a través de reuniones de capacitación y a su vez, implementar estrategias grupales

creando conciencia en la comunidad educativa. Este proceso de apoyo al estudiante migrante con las

herramientas de las TIC facilita los procesos de enseñanza -aprendizaje para la socialización integración y

seguridad con la regulación del delito cibérnetico.

Contreras Manrique, Rocío de Belén y Contreras Manrique, Liliana. (2014). Transversalidad de las TIC y prácticas pedagógicas en la educación actual Con la introducción de las tecnologías de la información y las comunicaciones en la enseñanza y el aprendizaje, a demás, la práctica pedagógica se ha visto transformada, pasando del uso de tableros, tizas y el cuaderno a cuadros, a la virtualidad, donde encontramos tablets, smart phones, conexión $\mathrm{Wi}-\mathrm{Fi}$, intercambio de archivos On-line, libros en PDF, conferencias a través de Video-llamadas, redes sociales, blogs y demás instrumentos que facilitan las relaciones en la educación, explico 
y describió la importancia de estas herramientas indispensables en la formación académica e integral de los educando y sobre todo en este tiempo de pandemia y dificultad social. Es relevante en las instituciones educativas la creación de ambientes virtuales apropiados para la enseñanza, el aprendizaje y su la retroalimentación para socializar los contenidos programáticos en cada asignatura. El ministerio de las TIC debería comprometerse en que las herramientas lleguen con facilidad en cualquier lugar para que la población migrante y los educandos colombianos obtengan el beneficio a la educación colombiana.

Arenas, Vivian, Silva, Gloria, Annicchiarico, Julio, Contreras, Liliana, Luna Ginny, Martìnez Yadira, Bermudez, Valmore, Jaime Yanela; Raynaud, Natalie.(2018). Tendencias en intervención psicológica y psicoterapia en el Norte de Santander. Tuvieron en cuenta aspectos genéricos como Psicoterapia e Intervención psicológica; así como los campos específicos educativo, clínico, social, organizacional, Jurídico, Forense y Salud. Se presenta una revisión de estudios de tipo cualitativo y cuantitativo, que generan aportes en: 1). Comprensión del estado psicológico de los habitantes de la región, 2). Análisis de variables psico-sociales relacionadas estado psicológico de la población nortesantandereana y 3). Se revisaron métodos terapéuticos y sus efectos en el mejoramiento de la salud mental.

Contreras-Manrique Liliana, ContrerasManrique Rocío de Belén, Ovalle-Lizcano Tatiana Valentina, Jaime Fernando RincónLeal. (2020). Análisis descriptivo de rasgos de personalidad en relación con el género y la edad de los estudiantes de la universidad de pamplona en la sede villa rosario. Los rasgos de personalidad que influyen en los estudiantes ya sea en su medio ambiente como en el nivel escolar, familiar y social, particularmente en los procesos de socialización con niveles de ansiedad, sentimientos de tristeza o trastorno como la depresión, agresividad que afectan al estudiante en su proceso de aprendizaje e interacción con su comunidad educativa, familiar y social

Contreras Manrique, L., Contreras Manrique, R. d., \& Ovalle Lizcano, T. V. (2019). Asociación Fonoaudiológica, Psicológica y Odontológica en la patología del Bruxismo: en los universitarios de Cúcuta. Los síntomas de la ansiedad psíquica que se presenta en los estudiantes. De tal manera, prevalecen los síntomas psíquicos como la escala de ansiedad con características de preocupaciones, tensión, insomnio y estrés. Estos sintomas, se presentan en los estudiantes migrantes venezolanos ya que se encuentra en dificultad escolar, económica, familiar y social.

Frias Ardila, Ruben Dario, Orduz, Jeison Alexander (2019). Percepción del nivel de autoconcepto y apoyo social asociadas a las características sociodemográficas en adolescentes escolares migrantes y nacionales de colegios públicos de la ciudad de Cúcuta, durante el segundo semestre de 2018 y el primer semestre 2019. Determino el nivel de autoconcepto y apoyo social relacionado con las características sociodemográficas de adolescentes escolarizados migrantes y nacional en seis colegios públicos de Cúcuta, en el estudio, no se evidencio relación entre autoconcepto, apoyo social y características sociodemográficas.

\section{METODOLOGÍA}

La investigación tiene como enfoque cuantitativo siguiendo la metodología de Hernández, Fernández y Baptista (2008), "el enfoque cuantitativo utiliza la recolección y el análisis de datos para contestar preguntas de investigación, confía en la medición numérica, el conteo y frecuentemente en el uso de estadística para establecer con exactitud patrones de comportamiento en una 
población" (p.5).En todo trabajo investigativo es necesario determinar el tipo de investigación que se aplicará; se busca se puede establecer el enfoque, para Hernández; Fernández \& Baptista (2008). la investigación cuantitativo no experimental. El diseño de la investigación es descriptivo, según Tamayo (2004) es aquella que comprende la descripción, registro, análisis e interpretación de la naturaleza actual, composición o procesos de los fenómenos. Este enfoque trabaja sobre las realidades de los hechos y sus características fundamentales, para presentarnos una interpretación correcta. (p. 54). El muestreo no probabilístico se realiza por el criterio, juicio y decisión del investigador para elegir los elementos de forma subjetiva o criterios no basados en el azar (Hurtado 2008), se encuentran el muestreo intencional o por conveniencia, teniendo en cuenta criterios teóricos que de alguna manera sugieren que ciertas unidades son las más convenientes para acceder a la información que se necesita. corregimiento de la Parada, Norte de Santander y se aplicara la escala TMMS-24 contiene tres dimensiones claves de la IE con 8 ítems cada unao de los siguientes factores: atención o percepción, clarida emocional o comprensión y feactor de reparación o regulación emocional. basada en Trait Meta-Mood Scale (TMMS) del grupo de investigación de Salovey y Mayer.

\section{RESULTADOS,ANÁLISISEINTERPRETACIÓN}

En la muestra de estudio con los estudiantes inmigrantes; en el factor de atención a nivel de percepción "Soy capaz de sentir y expresar los sentimientos de forma adecuada “, como respuesta de los estudiantes inmigrantes existe diferencia significativa en la inteligencia emocional. Un 60\% de los estudiantes migrantes, tienen el factor de atención; resaltando la expresión de sus sentimientos y un $40 \%$ de los estudiantes migrantes tienen ansiedad e inseguridad vivir en otro país. Se evidenció, un

$80 \%$ de los estudiantes migrantes, en el factor de claridad emocional, en la cual comprenden sus sentimientos y razonan emocionalmente; finalmente, el factor de reparación, la habilidad para regular emociones propias y ajenas con el nivel de regulación, los resultados demuestran, un $50 \%$ en los estudiantes migrantes tienen dificultades en regular o reparar los estados emocionales correctamente.

\section{Tabla I. Factores que inlfuyen en la Inteligencia Emocional}

\begin{tabular}{|l|c|}
\hline $\begin{array}{l}\text { Factor de Atención o } \\
\text { percepción }\end{array}$ & $60 \%$ \\
\hline $\begin{array}{l}\text { Factor de Claridad o } \\
\text { Comprensión Emocional }\end{array}$ & \\
\hline $\begin{array}{l}\text { Factor de Reparación o } \\
\text { regulación }\end{array}$ & $50 \%$ \\
\hline
\end{tabular}

Fuente Autores propios

\section{Figura 1. Factores que influyen en la inteligencia emocional}

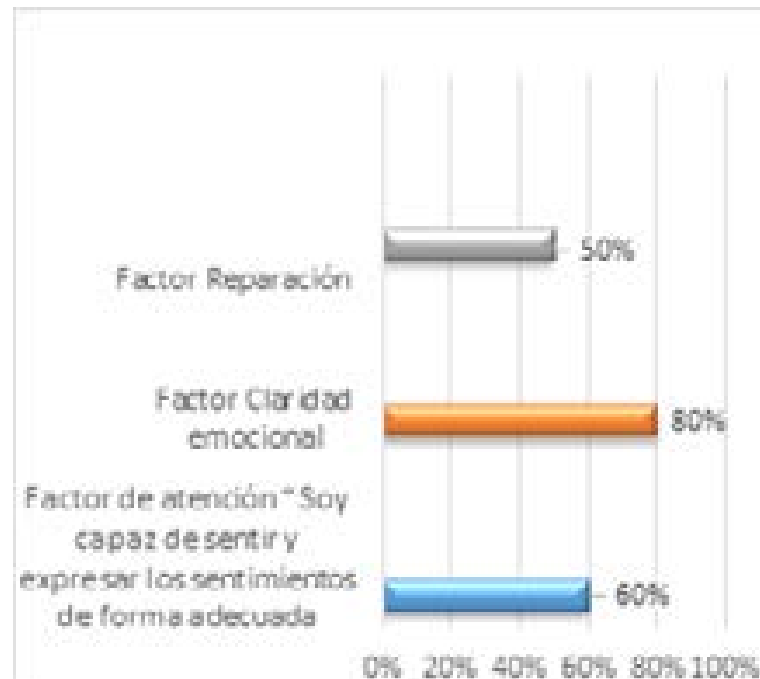

Fuente Autores propios 


\section{PROPUESTA DE MEJORAS}

Los estudiantes migrantes, en el proceso de la adaptación es fundamental, realizar encuentros formativos para fortalecer las habilidades personales como la autoestima, la autoimagen, el autoconcepto y la motivación; también se tienen en cuenta las actividades en las habilidades sociales como la comunicación asertiva, liderazgo e interacción a través de las interculturas con diferencias en métodologia en el aprendizaje.

En las mejoras del proceso del aprendizaje, se deben desarrollar, actividades en métodos y técnicas de estudio con herramientas de mapas mentales, mapas conceptuales, síntesis, ensayos, análisis e interpretación lectora.

Encuentros formativos de padres, profesores y estudiantes con la orientación de la psicóloga.

\section{DISCUSIONES}

$\begin{array}{llll}3 & 9 & 1 & \text { En los antecedentes; los autores Bustos, Raùl }\end{array}$ y Gairín Joaquín. (2017). Con la Investigación adaptación académica de estudiantes migrantes en contexto de frontera en Chile. Los resultados señalan que el contexto en estudio cuenta con una limitada predisposición a la interculturalidad. Por lo tanto, en la investigación; los educandos se encuentran con arraigo de la cultura venezolana, presentando su dificultad en la adaptación en los procesos educativos colombiano; siendo evidente en los colegios con la premisa de que es el estudiante, quien debe adaptarse al sistema escolar. En este caso es erroneo ya que los países deben garantizar la educación con igualdad y a su vez, que sea incluyente, siendo humana y originando la adaptación y estabilidad socioeducativo en un ambiente armonioso para desarrollar el potencial humano en los educandos migrantes. ( UNESCO, 2017, P7).

Es significativo, según los autores Salas, N., Castillo, D., San Martín, C., Kong, F., Thayer, E., \& Huepe, D. (2017). Reconocer el prejuicio que existente hacia los migrantes en el sistema escolar, tanto a nivel explícito como implícito, corroborando la hipótesis, se hace necesario una la propuesta psicosocial educativa para brindar orientaciones tendientes a potenciar la inclusión de la diversidad cultural en el sistema educacional, tanto a nivel de contexto, de políticas como de prácticas pedagógicas; siendo relevante el apoyo de los docentes, psicorientador y padres de familia en cada uno de los estudiantes en forma individual para los procesos de adaptación escolar. El Plan Nacional de desarrollo "Pacto por Colombia, pacto por la equdad (2018-2022); para mejorar la convivencia escolar e inclusión educativa en los niños y adolescentes migrantes provenientes de Venezuela. Desafortunadamente este beneficio de adaptación educativo y oportunidad de acompañamiento en el bienestar y permanencia del educando migrante, no ha tenido impacto en los educandos migrantes, siendo reflejado la falta de acompañamiento y fortalecimiento de la inteligencia emocional en los migrantes a través de la escala de TMMS-24 que se aplico en la investigación

Miranda Asenciocamilo Sebastián. (2019). Percepción de inclusión escolar que tienen los estudiantes migrantes de educación secundaria de establecimientos educacionales municipales en la ciudad de Concepción. Universidad del Desarrollo. Lo anterior, hace referencia a la urgencia y a la necesidad de saber cuál es la atención de inclusión que tienen los estudiantes migrantes para realizar procesos de resiliencia $y$ es lamentable, la falta de seguimiento $y$ orientación para cada educando migrante, sin embargo, esta contemplado en el Plan Nacional de desarrollo "Pacto por Colombia, pacto por la equdad (2018 -2022); para mejorar la convivencia escolar e inclusión educativa. 


\section{CONCLUSIONES}

En los estudiantes migrantes del corregimiento de la parada; el factor que influye en la inteligencia emocional en el corregimiento de la Parada Norte de Santander. Por lo tanto, los factores que presentan dificultad los estudiantes migrantes son los factores de atención o percepción y factor reparación o regulación de las emociones, ya que representa otra cultura y normatividad en ámbito social - educativo colombiano. Por tal motivo, se hace necesario implementar estrategias y seguimientos en base a la inteligencia emocional para mejorar su armonía social-educativo

\section{AGRADECIMIENTO}

estudiantes inmigrantes del corregimiento de la

Parada municipio de Villa Rosario

\section{RECOMENDACIONES}

Los docentes y el psicorientador debe ser el apoyo de los educandos migrantes; ya que, unas de las funciones, seria, el observar a los estudiantes cómo evolucionan en proceso de aprendizaje e interacción con otros pares; también, es recomendable que los estudiantes inmigrantes tengan un par al lado para que lo oriente en sus actividades escolares. Por lo tanto, es indispensable que los padres de familia y el titular del grupo sea el apoyo de los educandos; si embargo, el psicólogo del colegio, su función es asesor y fortalecer la personalidad, la convivencia con otros pares y orientar su proyecto de vida.

Apoyo a los estudiantes inmigrantes por parte de la secretaria de educación a travès del material educativo como guias, talleres, cuestionarios, lecturas, estableciéndose dentro de la normatividad educativa colombiana.

\section{REFERENCIAS BIBLIOGRÁFICAS}

Aravena Kenigs, Omar, Riquelme Bravo, Paula, Mellado Hernández, Ma Elena, \& Villagra Bravo, Carolina. (2019). Inclusión de Estudiantes Migrantes en la Región de La Araucanía, Chile: Representaciones desde los Directivos Escolares. Revista latinoamericana de educación inclusiva, 13(1), 55-71. https://dx.doi.org/10.4067/ $\underline{\text { S0718-73782019000100055 }}$

Arenas Villamizar, Vivian, Silva Monsalve, Gloria Inés, Annicchiárico Lobo, Julio Humberto, Contreras Manrique, Liliana, Luna Rodríguez, Ginny Roció, Martínez de Biava, Yadira, Bermúdez, Valmore, Jaimes Parada, Yanela Paola, Raynaud, Nathalie. Tendencias en intervención psicológica y psicoterapia en el Norte de Santander-Colombia Vol 37, No 5 AVFT - Archivos Venezolanos de Farmacología y Terapéutica, 2018.

Bastian Carter Thuillier, Víctor Manuel López Pastor, Francisco Javier Gallardo Fuentes. La Integración de los Estudiantes Inmigrantes en un Programa de Deporte Escolar con Fines de Transformación Social. Qualitative Research in Education Vol. 6 Núm. 1 Pág. 22-55. 2017.

Barahona, S. P. (2003). El estatuto de" Refugiado" en la Convención de Ginebra de 1951.

Revista electrónica del Departamento de Derecho de la Universidad de La Rioja,

REDUR, (1), 225-250. Recuperado de.https://dialnet.unirioja.es/servlet/ articulo? codigo $=624726$ 
Beniscelli, Leonora, Riedemann, Andrea, \& Stang, Fernanda. Multicultural $y$, sin embargo, asimilacionista. Paradojas provocadas por el currículo oculto en una escuela con alto porcentaje de alumnos migrantes. Calidad en la educación, (50), 393-423. 2019. https:// dx.doi.org/10.31619/caledu.n50.522

Bustos-González, R.. Estrategias de adaptación académica en estudiantes inmigrantes de establecimientos de enseñanza básica y media en la ciudad de Arica Dep. de Pedagogia Aplicada. PI. Coneixement (Edifici G6/247). 2016

Bustos, Raúl, \& Gairín, Joaquín.. Adaptación académica de estudiantes migrantes en contexto de frontera. Calidad en la educación, (46), 193-220. 2017. https://dx.doi.org/10.4067/s071845652017000100193. 2017.

Castillo, Dante, Santa-Cruz, Eduardo, \& Vega, Alejandro. Estudiantes migrantes en escuelas públicas chilenas. Calidad en la educación, (49), 18-49. 2018. https:// dx.doi.org/10.31619/caledu.n49.575. 2018.

Castillo Dante. Et al. Programa Interdisciplinario de Investigaciones en Educación PIIEMaría Luisa Santander 0440, Providencia -Santiago. 2016.

Cazzetta, Hannah María. Desafíos para los estudiantes venezolanos en Colombia. ESAL - Revista de Educación Superior en América Latina. 2019.

Diario la Opinión. https://www.laopinion.com.co/ frontera/el-caos-de-vivir-en-la-parada182596\#OP. 2019.
Contreras-Manrique Liliana, Contreras-Manrique Rocío de Belén, Ovalle-Lizcano Tatiana Valentina, Jaime Fernando RincónLeal. (2020). Análisis descriptivo de rasgos de personalidad en relación con el género y la edad de los estudiantes de la universidad de pamplona en la sede villa rosario. DOI https://doi. org/10.22463/17948231.2943.

Contreras-Manrique, R. de B., ContrerasManrique, L.., Ovalle-Lizcano, T. V.., Espinosa-Bohórquez, L. M. ValeroBencardino, J. ., Coronel-Peñuela, D. L. ., \& Cabrera-Reyes, A. (2020). Descripción de los problemas sociales influyentes en la transición moral en una sociedad cambiante a través del enfoque cuantitativo. DOI https://doi. org/10.22463/17948231.3016 .

Contreras Manrique, L., Contreras Manrique, R. d., \& Ovalle Lizcano, T. V. (2019). Asociación Fonoaudiológica, Psicológica y Odontológica en la patología del Bruxismo: En jóvenes universitarios de Cúcuta. Areté, 19 (1), [pgln]-[pgOut]. Obtenido de: https:// arete.ibero.edu.co/article/view/6586-1. DOI: 10.33881/1657-2513.art.19107

Durán, E. C., \& Parada, M. M. (2018). Obligaciones del estado respecto a la educación de los niños y niñas migrantes irregulares en el territorio nacional. Tesis de grado. Universidad Libre Seccional Cúcuta. Facultad De Derecho, Ciencia Política Y Sociales Especialización En Derechos Humanos. Recuperado de https://repository.unilibre.edu. co/bitstream/handle/10901/11713/ PAPER\%20DURAN\%20

PARADA\%20FINAL. pdf?sequence=1\&isAllowed=y 
Ferrer, Raquel; Palacio, Jorge; Hoyos, Olga and Madariaga, Camilo. Proceso de aculturación y adaptación del inmigrante: características individuales y redes sociales. Psicol. caribe [online]. 2014, vol.31, n.3, pp.557-576. ISSN 2011-7485. http://dx.doi.org/10.14482/ psdc.31.3.4766. 2014

Franco García, Martha JosefinaLos estudiantes inmigrantes: sujetos emergentes del derecho a la educaciónRevista Latinoamericana de Estudios Educativos (México), vol. XLIV, núm. 1, 2014, pp. 93-131Centro de Estudios Educativos, A.C.Distrito Federal, México.2014.

Frias Ardila, Ruben Dario, Orduz, Jeison Alexander Percepción del nivel de autoconcepto y apoyo social asociadas a las características sociodemográficas en adolescentes escolares migrantes $y$ nacionales de colegios públicos de la ciudad de Cúcuta. UFPS. (2019)

Goleman, D. Y Otros. Vergara, Editor. Bs. Aires. Argentina. 2010

Goleman, D. La Inteligencia Emocional. Vergara, Editor. Bs. Aires. Argentina. Goleman, D. Y Otros. 2000. Vergara, Editor. Bs. Aires. Argentina . 2000

Hernández, R; Fernández, C. y Baptista, P. Metodología de la Investigación. Bogotá: McGraw Hill. 2008

Hurtado de Barrera, J. TerceraEdición. Fundación Sypal: Caracas. 2010. (Parte IICapítulo 3 y 4).

Manzo Álvarez, Claudia Libertad. Perspectivas y prácticas vinculadas a inclusión presentes en la evaluación de desempeño de establecimientos y sus sostenedores: Análisis cualitativo de informes de visitas evaluativas.
Universidad Alberto Hurtado Facultad de Educación, 2018.

Miranda Asencio Camilo Sebastián. Percepcióndeinclusión escolar que tienen losestudiantes migrantes de educación secundaria de establecimientos educacionales municipales en la ciudad de Concepción. Universidad del Desarrollo. Facultad de GobiernoMagíster en Políticas Educativas. Concepción. 2019

Mondaca, Carlos, Muñoz, Wilson, Gajardo, Yeliza, \& Gairín, Joaquín. Estrategias y prácticas de inclusión de estudiantes migrantes en las escuelas de Arica y Parinacota, frontera norte de Chile. Estudios atacameños, (57). 2018 181201. https://dx.doi.org/10.4067/S071810432018005000101.

Mondaca Rojas, Carlos Enrique. Educación y migración transfronteriza en el norte de chile: procesos de inclusión y exclusión de estudiantes migrantes peruanos y bolivianos en las escuelas de la región de arica y Parinacota. 2018.

Nogueira, A. (2017). La protección convencional de los Derechos de los Niños y los estándares de la Corte IDH sobre medidas especiales de protección por parte de los estados parte respecto de los niños, como fundamento para asegurar constitucionalmente los Derechos de los Niños y Adolescentes. lus et Praxis, 23(2),415-462. Recuperado de https://scielo.conicyt.cl/scielo.php? S071800122017000200415 
López Villamil, S., Rodríguez Lizarralde, C., González, L. D. A., \& Barriga Durán, L. C. (2018). Infancias migrantes en Colombia: retos del derecho a la educación. ojas ablas, (16), 10-26. https://doi.org/10.29151/hojasyhablas. n16a1

Salas, N., Castillo, D., San Martín, C., Kong, F., Thayer, E., \& Huepe, Inmigración en la escuela: caracterización del prejuicio hacia escolares migrantes en Chile. Universitas Psychologica, 16(5), 1-15. https://doi.org/ 10.11144/Javeriana. upsy16-5.iecp. 2017.

Salovey, P., Mayer, J. D., Goldman, S. L., Turvey, C., Palfai, T. P. Emotional attention, clarity, and repair: exploring emotional intelligence using the Trait Meta-Mood Scale. En J. W. Pennebaker (Ed), Emotion, Disclosure, y Health (pp. 125-151). Washington: American

Serna M. E., y Serna A, A. Laformación en ingeniería en Colombia: una situación que preocupa. 2013.

Sosa Fernanda Mariel y Zubieta Elena. la experiencia de migración y adaptación sociocultural: identidad, contacto y apoyo social en estudiantes universitarios migrantes. psicogente [ONLINE]. 2015, vOL.18, n.33, pP.3651. issn 0124-0137. hTTP://dX.dOI. oRG/10.17081/pSICO.18.33.54. 2015

Suárez, A; Espinel, M; Contreras Manrique, L; Contreras Manrique, R. (2015). Herramientas de apoyo para el aprendizaje de los estudiantes que presentan limitaciones auditivas. Revista Areté, Editor Corporación Universitaria Iberoamericana, volumen (15), 1. 52-62. ISSN 1657-2513. DOI: 10.33881/16572513

Ramos, N., Enríquez, H. y Recondo, O. Inteligencia Emocional Plena. Barcelona: Kairós.2012

Ruiz Monterroso, lleana Ninette. Los Niveles de las dimensiones de inteligencia emocional en los alumnos de primero y quinto año de las carreras de psicología clínica e industrial de la jornada vespertina. guatemala de la asunción. Universidad Rafael Landivvar. Facultad De Humanidades. Maestría En Docencia De La Educación Superior. 2014.

RBC Manrique, LC Manrique - Cuadernos de Educación y Desarrollo. Transversalidad De Las Tic Y Prácticas Pedagógicas En La Educación Actual. Grupo Eumed. net . Universidad de Málaga. 2014

Rincón Peña. Diego Hernán. (2019). El derecho a la educación de los niños, niñas y adolescentes migrantes venezolanos desde la perspectiva de los Derechos Humanos. Facultad de Derecho de la Universidad Católica de Colombia.

Tamayo y Tamayo. El Proceso de la Investigación. México: Limusa. p. 54. 2004

TVO Lizcano, DLC Peñuela, RBC Manrique, AC Reyes - revista Respuestas, 2019. Dialnet

Torres, E. (2019). Derechos Humanos Y Estrategias De Encubrimiento $Y$ Legitimación En Discursos Del Instituto Nacional De Migración Sobre La Transmigración Por México (Human Rights and Strategies of Concealment and Legitimization in the National 
Institute of Migration Discourse on Transit Migration). Revista Mexicana de Ciencias Políticas y Sociales, 64(237). Recuperado de https://papers.ssrn.com/ sol3/papers.cfm?abstract_id $=3445104$

UNESCO. (2019). Derecho a la educación | Organización de las Naciones Unidas para la Educación, la Ciencia y

la Cultura. Recuperado el

20 de septiembre 2019 , de

http://www.unesco.org/new/es/social-andhumansciences/themes/advancement/ networks/larno/right-to-education/

UNICEF. (2016). Protocolo de evaluación inicial para la identificación de indicios de necesidades de protección internacional en niñas, niños y adolescentes no acompañados o separados.

Ciudad de México. Recuperado de

https://www.unicef.org/mexico/media/1216/file/ Protocolo_Proteccion_Especial_CO MAR.pdf

UNICEF. (2016). Protocolo de evaluación inicial para la identificación de indicios de necesidades de protección internacional en niñas, niños y adolescentes no acompañados o separados.

Ciudad de México. Recuperado de https://www.unicef.org/mexico/media/1216/file/ Protocolo_Proteccion_Especial_CO MAR.pdf

\section{TRATADOS, CONVENIOS Y NORMAS}

Congreso de la República de Colombia. (2006) Ley 1098 de noviembre 8 "Por la cual se expide el Código de la Infancia y la Adolescencia". Diario Oficial No. 46.446 de 8 de noviembre de 2006.

Pacto Internacional de Derechos Económicos, Sociales y Culturales. (1966).

Asamblea General de las Naciones Unidas. Resolución 2200 A (XXI), de diciembre 16.

\section{JURISPRUDENCIA}

Corte Constitucional de Colombia (2013) Sentencia T-743 de octubre 23. M.P Luis Ernesto Vargas Silva.

Corte Constitucional de Colombia (2017) Sentencia SU-677 de noviembre 15. M.P Gloria Stella Ortiz Delgado.

https://caracol.com.co/emisora/2020/04/20/ cucuta/1587391271_082238.html. 2020

https://www.elespectador.com/noticias/elmundo/philip-goldberg-confirmadocomo-nuevo-embajador-de-eeuu-encolombia-articulo-873938.2019

\section{https://www.laopinion.com.co/frontera/ adaptacion-escolar-prueba-para- inmigrantes-174232\#OP. 2018}

